\title{
NA CONTRAMÃO DA INFORMAÇÃO PREVENTIVA: DESINFORMAÇÃO SOBRE PREVENÇÃO DE HIV/AIDS
}

\author{
Tereza Evâny de Lima Renôr Ferreira \\ Doutoranda em Ensino das Ciências \\ Professora do Curso de Administração da UFRPE \\ terezarenor@yahoo.com.br \\ Júlio Afonso Sá de Pinho Neto \\ Doutor em Comunicação e Cultura \\ Departamento de Comunicação/ PPGCI/UFPB e MPGOA/UFPB \\ sadepinho@uol.com.br
}

Resumo

\begin{abstract}
O acesso e o uso da informação pelos jovens, nos dias atuais, e os números ainda relevantes de casos de contaminação provenientes do vírus HIV motivaram o desenvolvimento desta pesquisa para se conhecer o nível de desinformação dos jovens na prevenção do HIV/AIDS, já que, segundo o Programa Conjunto das Nações Unidas sobre HIV/AIDS (UNAIDS), a desinformação sobre o assunto é a grande causa de jovens contraírem o HIV. Contrariamente à informação, a desinformação remete o indivíduo a um estado de ignorância em relação ao conhecimento que lhe seria relevante. Portanto a informação tem a função de orientá-lo e de torná-lo capaz de participar do processo cognitivo e evolutivo da cadeia informacional e social. O objetivo desta pesquisa foi de conhecer o nível de desinformação sobre a prevenção do HIV/AIDS entre os jovens, considerando as estratégias de prevenção combinada do HIV/AIDS. Neste estudo, caracterizado como uma pesquisa exploratória, foi empregada a técnica de snowball para a coleta dos dados. Seus resultados foram configurados em gráficos e tabelas para uma posterior análise quantitativa e qualitativa. Os resultados apontaram que o nível de desinformação sobre a prevenção do HIV/AIDS entre os jovens pesquisados é baixo, porque, das questões respondidas, apenas três apresentaram relação com a desinformação. Isso significa que, apesar do acesso à informação, a informação preventiva é negligenciada.
\end{abstract}

Palavras-chave: Desinformação. Informação. Prevenção do HIV/AIDS.

\section{INTRODUÇÃO}

Este trabalho traz um estudo a respeito da desinformação sobre a prevenção do HIV/AIDS, considerando os riscos e as vulnerabilidades para contrair o vírus, sobretudo quando se disponibilizam para a população jovem canais e acesso à informação, um público que tem uma relação íntima com os diversos formatos e veículos de informação. Segundo o Programa Conjunto das Nações Unidas sobre HIV/AIDS (UNAIDS), especula-se que a desinformação sobre o assunto é a grande causa de jovens contraírem o HIV, e na América Latina, o número de pessoas com o problema ainda é alto. "Uma dolorosa maioria de adolescentes e jovens não têm informação sobre como evitar o contágio, inclusive em países onde a enfermidade está propagando-se rapidamente e alcançando níveis de pandemia", assim divulgou o Fundo das Nações Unidas para a Infância - UNICEF (2010). Apesar de tantas informações disponibilizadas, a desinformação é uma realidade que mata e destrói.

A desinformação, concebida como a "ausência de informação", é relacionada a um estado de ignorância do indivíduo em relação ao conhecimento relevante para seu desenvolvimento e sua atuação na sociedade 
(NEHMY; PAIM, 1998; AQUINO, 2007). Já a desinformação, entendida como a "informação manipulada", remete a outra compreensão, relacionada ao fornecimento de produtos informacionais de baixo nível cultural, cuja consequência direta seria a "imbecilização" de setores sociais (DEMO, 2000). Nesta pesquisa, foi considerado o primeiro conceito, relacionado à falta de informação, para fins preventivos, visando evitar o contato com o vírus. Para isso, objetivou conhecer o nível de desinformação sobre a prevenção do HIV/AIDS entre os jovens, porquanto o acesso à informação e seu uso têm conseguido otimizar a comunicação dos dias atuais.

Para estruturar a pesquisa, recorreu-se às estratégias de prevenção combinada do HIV/AIDS, destinadas a profissionais, a trabalhadores/as e a gestores/as de saúde e dos movimentos sociais para combater a epidemia do Vírus da Imunodeficiência Humana (HIV) e da Síndrome da Imunodeficiência Adquirida (AIDS), produzida pelo Ministério da Saúde, mediante a Secretaria de Vigilância, Prevenção e Controle das Infecções Sexualmente Transmissíveis do HIV/AIDS. Essas estratégias de prevenção de uso combinado são intercaladas por meio das intervenções biomédicas, comportamentais e estruturais aplicadas aos indivíduos, de suas relações e dos grupos sociais a que pertencem, com ações que levem em consideração suas necessidades e especificidades e as formas como o vírus é transmitido (BRASIL Secretaria de Vigilância, 2017).

Para produzir este artigo, foram adotadas a teoria sobre a desinformação e estratégias de prevenção sobre HIV/AIDS. Em seguida, foram expostos os caminhos metodológicos percorridos para consolidar a pesquisa, seguida das análises e das referências.

\section{INFORMAÇÃO E DESINFORMAÇÃO}

Para entender o conceito de 'desinformação', é necessário entender o de 'informação', visando balizar os laços sobre um termo e outro. A informação conduz, direciona e aponta caminhos antes nunca percorridos para quem passa a usá-la, além de ser o ponto de partida para dar início ao processo cognitivo, que produz significado por meio da aprendizagem, para se adquirir o conhecimento. Segundo González de Gómez
(1999), a informação atua em um processo contínuo, porquanto o sujeito tem competência para produzir novas informações por meio do acesso a ela, do seu uso e de sua apropriação, modificando os meandros da sociedade por meio da interferência informacional. A informação tem um caráter social produzido a partir das necessidades cotidianas, implementadas nos processos informacionais da sociedade, capazes de modificar o comportamento das pessoas, comprometendo sua forma de agir na sociedade.

$\mathrm{Na}$ contramão da informação, está a informação que desinforma, que confunde e que desorienta, que remete ao ruído ou à falta de informação e dá margem à alienação nos indivíduos sociais. Para Pinheiro e Brito (2014), a falta de informação desencadeia a desinformação baseada na falta de cultura ou de competência informacional, que incapacita o usuário a localizar a informação e impossibilita a mudança de um sujeito sem orientação para um sujeito com orientação. Essa temática está relacionada ao nível de cognição do sujeito e, consequentemente, ao seu nível de conhecimento, uma vez que a informação faz parte do processo de interpretação para uma posterior mudança em sua estrutura mental e social. Sobre a falta de acesso à informação, Aquino (2007) busca relacionar o conceito de desinformação aos ruídos e às abundâncias e afirma que o acesso à informação compartilhada pelo mundo reduz a possibilidade de acesso e de uso da informação quando $o$ sujeito não tem capacidade de usar a informação globalizada, seja pelo excesso ou pela falta de interesse, e os usuários recebem e compartilham informação de valor cultural baixo ou sem nenhuma utilidade e deixam de lado a informação que modifica e agrega conhecimento ao contexto social.

\section{POLÍTICAS PÚBLICAS DE SAÚDE}

\subsection{PREVENÇÃO COMBINADA DO HIV}

O Brasil, por meio de políticas públicas para o combate ao HIV/AIDS, tem recorrido a vários dispositivos e parceiros que $\mathrm{o}$ diferenciam de outras realidades no tocante à prevenção e ao tratamento de pessoas vulneráveis e infectadas. A prevenção combinada do HIV/AIDS é uma estratégia de prevenção em que se usa um combinado de intervenções biomédicas, comportamentais e 
estruturais aplicadas no nível dos indivíduos, de suas relações e dos grupos sociais a que pertencem, por meio de ações que levem em consideração as necessidades, as especificidades e as formas de transmissão do vírus (MINISTÉRIO DA SAÚDE, p. 9, 2017).

A Prevenção Combinada do HIV associa ações estratégicas e conjuntas de prevenção do vírus HIV e os fatores associados à infecção. Para tanto, foram formuladas três ações baseadas nas intervenções biomédicas, comportamentais e estruturais, direcionadas aos grupos sociais a que pertencem, levando em consideração as necessidades, as especificidades e as formas como o vírus é transmitido (BRASIL - Secretaria de Vigilância, p.18, 2017).

Os quadros abaixo apresentam os 'Componentes da prevenção combinada do HIV' e as categorias representadas pelas intervenções biomédicas, comportamentais e estruturais, bem como, as definições das intervenções e as ações preventivas.

Quadro 1 - Componentes da prevenção combinada do HIV - Intervenções biomédicas

\begin{tabular}{|c|l|}
\hline Definição & \multicolumn{1}{c|}{ Ações preventivas } \\
\hline & - Preservativo feminino e masculino, associados a gel \\
& lubrificante; \\
São estratégias voltadas para reduzir o & - Tratamento para todas as pessoas - TTP; \\
risco de exposição ou de & - Profilaxia pós-exposição - PEP; \\
transmissibilidade com o uso de & - Profilaxia pré-exposição - PrEP; \\
antirretrovirais ou de outras tecnologias & - Prevenção e tratamento das infecções sexualmente \\
biomédicas. & transmissíveis - IST; \\
& - Imunização para hepatite B e HPV; \\
& - Prevenção da transmissão vertical. \\
\hline
\end{tabular}

Fonte: Departamento de Vigilância, Prevenção e Controle das IST, do HIV/AIDS e das Hepatites

Virais - 2016.

As intervenções biomédicas são voltadas para reduzir os riscos de HIV. Essas ações visam bloquear a transmissão do vírus entre pessoas por meio das relações sexuais desprotegidas e de material biológico contaminado por HIV. Tais procedimentos são divididos em: intervenções biomédicas clássicas e antirretrovirais (ARV). Nas biomédicas clássicas, são visualizadas as barreiras físicas, como os preservativos. Já o ARV considera a profilaxia pré-exposição (PPE) e a profilaxia pós-exposição (PEP).

Quadro 2 - Componentes da Prevenção Combinada do HIV - Intervenções comportamentais

\begin{tabular}{|c|l|}
\hline Definição & \multicolumn{1}{c|}{ Ações preventivas } \\
\hline & - Adesão ao uso de preservativos masculino e feminino e de \\
& gel lubrificante; \\
& - Aconselhamento sobre HIV, hepatites virais e outras IST \\
(abordagem sobre redução e gerenciamento de risco e sobre \\
as possibilidades em determinado momento e contexto da \\
São estratégias que contribuem para o \\
aumento da informação e do \\
vida, fortalecendo a autonomia do indivíduo); \\
conhecimento e para se perceber ou & - Incentivo à testagem; \\
autoavaliar o risco à exposição ao HIV, & - Adesão às intervenções biomédicas; \\
o que colabora efetivamente para & - Vinculação e reteção nos serviços de saúde; \\
reduzir esse risco, com o incentivo a & - Reduçáo de danos para as pessoas que usam álcool e outras \\
mudanças de comportamento & drogas; \\
individual ou comunitário. & - Redução de danos para as pessoas que usam silicone \\
& industrial e hormônios; \\
& - Estratégias de comunicação e educação entre pares e \\
& fortalecimento das ações de base comunitária; \\
& - Campanhas de prevenção de HIV, hepatites virais e outras \\
\hline
\end{tabular}

Fonte: Departamento de Vigilância, Prevenção e Controle das IST, do HIV/Aids e das Hepatites Virais, 2016. 


\begin{tabular}{lll}
\hline No caso das intervenções & "grupo de risco", que apresenta fatores \\
comportamentais, o foco consiste em evitar & informacionais e cognitivos negligenciados \\
situações de riscos/vulnerabilidade por meio & em suas ações preventivas. Em saúde pública, \\
de informações e conhecimentos. As & o termo risco mantém a ideia central de \\
informações sobre a exposição do uso de & probabilidade, e o termo epidemiologia \\
álcool a vulnerabilidade de praticar sexo e & refere-se à probabilidade de um indivíduo, \\
usar drogas sem orientação devem ser & determinada população ou grupo específico \\
disseminadas para a população de forma & serem acometidos por uma doença, sofrerem \\
generalizada. A vulnerabilidade está & agravo, óbito ou outra condição relacionada à \\
associada diretamente ao comportamento do & saúde (WALDMAN, 1998).
\end{tabular}

Quadro 3 - Componentes da prevenção combinada do HIV- Intervenções estruturais

\begin{tabular}{|c|l|}
\hline Definição & \multicolumn{1}{c|}{ Ações preventivas } \\
\hline São estratégias voltadas para enfrentar & - Ações de enfrentamento ao estigma e à discriminação, \\
fatores e condições socioculturais que & como racismo, sexismo, machismo, homofobia, transfobia, \\
influenciam diretamente a & lesbofobia e outras formas de exclusão; \\
vulnerabilidade de indivíduos ou & - Promoção e defesa dos direitos humanos; \\
grupos sociais específicos, que sofrem & - Políticas afirmativas de garantias de direitos; \\
preconceito, estigma, discriminação, & - Diminuição das desigualdades socioeconômicas; \\
violência ou qualquer outra forma de & - Campanhas educativas e de conscientização. \\
alienação dos direitos e das garantias & \\
fundamentais à dignidade humana. & \\
\hline
\end{tabular}

Fonte: Departamento de Vigilância, Prevenção e Controle das IST, do HIV/AIDS e das Hepatites Virais -2016.

As intervenções estruturais visam mudar as causas ou estruturas centrais que afetam riscos e vulnerabilidades dos indivíduos ao HIV por meio de suas características sociais, culturais, políticas e econômicas. Essa intervenção visa suprimir preconceitos e discriminação que possam infringir direitos e garantias à dignidade humana e social.

As três intervenções são importantes e se complementam para atingir as populaçõesalvo. Para esta pesquisa sobre desinformação, foi escolhida a intervenção comportamental, por se tratar de uma abordagem que leva em consideração o compartilhamento de informações como uma forma eficaz de prevenir o HIV/AIDS.

\subsection{POPULAÇÕES-ALVO}

Para desenvolver e direcionar tais estratégias, o Ministério da Saúde classificou o público vulnerável em população chave e população prioritária, visando estruturar melhor esse trabalho. No Brasil, a epidemia de HIV é concentrada em alguns segmentos populacionais que, muitas vezes, estão inseridos em contextos que aumentam suas vulnerabilidades (população chave) $\mathrm{e}$ apresentam prevalência superior à média nacional, que é de 0,4\% (BRASIL, 2016), como: gays, pessoas trans, que usam álcool e outras drogas, privadas de liberdade e trabalhadoras do sexo.

A população jovem, caracterizada como população prioritária, envolve a população negra, a indígena e a de situação de rua. Apesar de o governo dividir a população em segmentos, eles não se sobrepõem entre si. Essa é apenas uma forma de organizar as intervenções, munidas de suas diferentes estratégias. É preciso entender que o aspecto de transversalidade vai muito além do recorte etário ou do quesito raça/cor das populações mais afetadas pelo HIV/AIDS. Todas as categorias entendidas como chave e prioritárias na resposta à epidemia devem ser consideradas como potencialmente transversais e fluidas. Assim, um gay pode usar drogas e fazer trabalho sexual; uma mulher transexual pode fazer trabalho sexual e estar ou ser privada de liberdade, e assim por diante. Dessa forma, o pertencimento a uma categoria não exclui a pessoa das demais, e as vulnerabilidades dessas populações devem ser entendidas em sua integralidade (BRASIL - Secretaria de Vigilância, 2017).

\section{CAMINHOS METODOLÓGICOS}

A pesquisa de cunho exploratório objetivou conhecer o nível de desinformação de jovens sobre a prevenção de HIV/AIDS. 
Para tanto, foi elaborado e aplicado um questionário on-line, com questões baseadas na Prevenção Combinada do HIV/AIDS, e empregada a intervenção comportamental, com jovens de 15 a 29 anos de idade, na cidade de João Pessoa/PB, uma das "populações prioritárias" classificada pelo Manual. Essa intervenção é uma das três adotadas pelo Manual de Prevenção de HIV/AIDS, criado pelo Ministério da Saúde Federal - Secretaria de Vigilância em Saúde. $\mathrm{Na}$ aplicação do questionário, foi empregada a técnica snowball (bola de neve), criada por Goodman (1961), uma das técnicas utilizadas em pesquisa social, em que os participantes iniciais indicam novos participantes, e os novos indicam outros que apresentem o perfil descrito até que o número de participantes seja alcançado (ALBUQUERQUE, 2009). Portanto, a snowball pode ser entendida como uma técnica de amostragem em que se utilizam cadeias de referência, caracterizada como uma rede e configurada como não probabilística por conveniência. Foram aplicados 86 questionários, compartilhados com três jovens estudantes universitários, alunos do Curso de Administração, denominados de 'sementes', um termo utilizado por Albuquerque (2009) referente ao maior número de pessoas do mesmo perfil pesquisado.

A pesquisa de cunho quantitativo trabalhou com percentuais, apontou o número de jovens desinformados sobre o tema $\mathrm{e}$ apresentou uma análise qualitativa, uma vez que se analisou o conteúdo produzido por meio de gráficos e de tabelas elaboradas a partir de questionários on-line, enviados pelo WhatsApp Messenger, um aplicativo de mensagens instantâneas configurado para smartphones, na cidade de João Pessoa/PB, no período de 29 de dezembro de 2017 a 05 de janeiro de 2018.

\section{ANÁLISE DOS RESULTADOS DA PESQUISA}

As quatro primeiras questões se reportaram ao levantamento do perfil demográfico. No que diz respeito ao perfil dos indivíduos pesquisados, foram constatados os seguintes resultados: a maioria é de jovens com faixa etária de 19 a 22 anos (51,2\%); 25,6\%, de 23 a 26 anos; $14 \%$, de 27 a 29 anos; e 9,3\%, de 15 a 18 anos. Percebese que as faixas etárias que vão de 19 a 26 anos, caracterizadas pelo maior número de respondentes, coincidem com a de maior número de pesquisados com ensino superior completo e incompleto. Esses dados estão relacionados a jovens que, além de já ter acesso à informação $(45,3 \%)$, cursaram o ensino superior incompleto, e $25,6 \%$, o ensino superior completo, portanto, apresentam um grau de maturidade para vivenciar uma vida sexual ativa, o que, para a pesquisa, é um percentual considerável, em se tratando de um público que tem acesso e discernimento para usar e buscar informações relevantes sobre a prevenção de HIV/AIDS.

A cor preponderante declarada por $46,5 \%$ dos pesquisados foi a parda, e a branca, por $45,5 \%$. Apenas $4,7 \%$ se declaram pretos. Foi questionada também a renda familiar, com o intuito de conhecer as condições econômicas dos pesquisados. Os resultados apontaram que $46,5 \%$ percebem uma renda familiar de um a três salários mínimos; $27,9 \%$, de quatro a seis salários mínimos; e apenas $10,5 \%$, de sete a nove salários mínimos.

$\mathrm{Na}$ sequência, foram elaboradas quatorze questões baseadas nas ações preventivas das intervenções comportamentais, cujo teor visa pesquisar o nível de desinformação da população prioritária, em especial, dos jovens de 15 a 29 anos de idade. As intervenções comportamentais visam reduzir o risco, levando em consideração a mudança de comportamento individual e social.

Os jovens pesquisados foram questionados, inicialmente, sobre se tinham o vírus da imunodeficiência humana (HIV) ou síndrome da imunodeficiência adquirida (AIDS). De acordo com suas respostas, nenhum (100\%) tem o referido. É importante pontuar que esse número não indica, necessariamente, que, por não estarem infectados, estão fora da zona de risco/vulnerabilidade para adquirir o vírus. Por isso, precisam continuar buscando e usando informações preventivas.

Quanto à questão 02, em que os sujeitos da pesquisa foram perguntados sobre se recorriam a informações a respeito do uso de preservativos masculino e feminino e de gel lubrificante, o nível de desinformação foi de 3,5\% negativo, ou seja, 96,5\% conhecem os preservativos mencionados na pesquisa. De acordo com o Manual Preventivo do Ministério da Saúde, 
[...] segundo os dados da última Pesquisa de Conhecimentos, Atitudes e Práticas na População Brasileira - PCAP 2013 (BRASIL, 2016b), a larga maioria dos brasileiros (94\%), incluindo os jovens, sabe que o preservativo é melhor forma de prevenção às IST, HIV e AIDS. A despeito disso, de acordo com a mesma pesquisa, quase metade $(45 \%)$ da população sexualmente ativa do país reportou não ter feito uso do preservativo (masculino ou feminino) nas relações sexuais casuais que tiveram nos 12 meses anteriores à pesquisa. (MINISTÉRIO DA SAÚDE, p.69, 2017).

O grupo pesquisado tem grandes chances de usar um dos procedimentos considerado simples e eficaz no combate ao vírus HIV.

A informação concedida por meio de conselhos de especialistas ou familiares sobre as possibilidades de se contrair o HIV é uma prática válida. No que diz respeito à questão 3 - sobre se os jovens receberam algum tipo de aconselhamento a respeito de HIV, hepatites virais e outras infecções sexualmente transmissíveis - IST, com abordagem sobre redução e gerenciamento de risco e as possibilidades de, em determinado momento e contexto da vida - 80,2\% dos jovens afirmaram que receberam esse tipo de aconselhamento, e 19,8\% não o receberam.

$\mathrm{Na}$ questão 04, os sujeitos da pesquisa foram perguntados sobre se, em algum momento, foram incentivados a fazer o teste HIV. A pesquisa mostrou que $64 \%$ não foram incentivados, e $36 \%$ foram incentivados. Esse percentual de $36 \%$ merece uma reflexão sobre a necessidade de se fazer o teste, caso mantenha vida sexual ativa com diversos parceiros, porque o teste pode revelar a presença do vírus e impedir que o indivíduo possa contaminar outros indivíduos. De acordo com a Pesquisa de Conhecimentos, Atitudes e Práticas - PCAP 2013, 36, 1\% dos indivíduos sexualmente ativos, com idades entre 15 e 64 anos fizeram o teste de HIV alguma vez na vida.

Essa proporção muda de acordo com variáveis sociodemográficas como idade, escolaridade, raça/cor ou classe social. Em relação à frequência com que as pessoas se testaram, a mesma pesquisa identificou que $63,9 \%$ revelaram nunca ter se testado e que apenas $10,2 \%$ haviam se testado uma vez no ano anterior, sendo ainda menor a proporção de pessoas que haviam se testado mais de uma vez no ano anterior: somente $3,2 \%$ da amostra pesquisada. (BRASIL, 2016b)

Foi questionado (questão 05) se os pesquisados mantiveram relação sexual com alguém que tinha dúvidas sobre se estava infectado. O estudo mostrou que 93\% afirmaram não ter dúvidas sobre seus parceiros nesse sentido, e $7 \%$ tiveram dúvidas sobre eles. Esse é um percentual de segurança elevado, mas não impede que os preservativos e os testes sejam utilizados para prevenir não somente HIV, mas também IST.

Como forma de profilaxia no contágio pelo vírus da hepatite $B$ e Human Papilomavirus - HPV, os sujeitos pesquisados foram perguntados sobre se são imunizados. De acordo com os resultados, $57 \%$ dos indivíduos disseram que foram imunizados, e $47 \%$, não. Tanto a hepatite B quanto o HPV são vírus que merecem cuidados, pois o papilomavírus humano tem mais de 200 variações e se manifesta por meio de formações verrugosas - que podem aparecer no pênis, na vulva, na vagina, no ânus, no colo do útero, na boca ou na garganta. O sexo é a principal forma de transmissão do HPV, seja pelo coito ou pelo sexo oral. O HPV é uma preocupação grave de saúde pública, porque alguns tipos de vírus têm um grande potencial para causar câncer, principalmente no colo do útero, no ânus, na boca e na garganta, que vêm aumentando entre os jovens (BRASIL - Secretaria de Vigilância em Saúde, 2017b).

A questão 07 procurou saber se os pesquisados sabiam como é feito o teste HIV. Essa informação é relevante, porque conviver com a dúvida é ter a possibilidade de transmitir o vírus ou não tomar as medicações indicadas para não contrair AIDS. Nesse sentido, 64\% sabem como o teste é feito, e $36 \%$ não têm acesso a esse tipo de informação.

A busca por informações é uma prática que faz parte da competência informacional dos usuários da informação. Pinheiro e Brito (2014) afirmam que a incompetência informacional incapacita o usuário a localizar a informação que o orienta e o transforma. $\mathrm{Na}$ questão 08, o estudo visou avaliar o nível de desinformação, que foi inferior $(24,4 \%)$ ao nível de informação, pois $75,6 \%$ afirmaram saber onde buscar informações sobre 
prevenção dos serviços a respeito do HIV/AIDS.

A questão 09 indicou que o nível de desinformação sobre profilaxia pré-exposição é alto, pois $73,3 \%$ nunca ouviram falar a seu respeito, e apenas $26,7 \%$ ouviram falar. A PrEP consiste em utilizar medicamentos antirretrovirais -ARV por pessoas HIV negativas para evitar que contraiam o vírus. A PrEP é altamente efetiva para prevenir o HIV quando utilizada corretamente. Por isso saber como se usa o medicamento é substancial para quem precisa consumi-lo.

No que diz respeito a ter acesso a informações sobre os riscos de os efeitos do consumo de drogas/álcool possibilitarem uma infecção do HIV e de levar a uma relação sexual com desconhecido infectado, a questão 10 revelou que $80,2 \%$ afirmaram ter acesso a esse tipo de informação, e 19,8\% disseram que não têm esse tipo de informação. $\mathrm{O}$ uso de drogas, sobretudo do álcool, é um componente importante da produção de masculinidades hegemônicas e compõe um conjunto de práticas que concorrem para situações de exposição ao HIV (MORAES; MONTENEGRO, 2014, GRANJA et al., 2015). Reforçando a pauta, a Portaria GM/MS $\mathrm{n}^{\mathrm{o}} 1.028 / 2005$ frisa a importância das ações de informação, educação e aconselhamento ao (à) usuário (a), em relação aos produtos, às substâncias ou drogas que causem dependência e às práticas sexuais das pessoas que usam álcool e outras drogas e suas parcerias, com o objetivo de estimular a adoção de comportamentos mais seguros tanto no consumo de substâncias psicoativas como nas práticas sexuais.

Em relação ao uso do silicone industrial e de hormônios, Mello et al. (2013) também recomendam que não se devem compartilhar seringas e usar agulhas descartáveis, para evitar a contaminação pelo HIV, hepatites virais e outras infecções (incluindo as causadoras de abscessos cutâneos). Na questão 11 - sobre se os jovens pesquisados têm informações sobre os riscos de infecção pelo HIV que as pessoas que usam silicone industrial e hormônios e não fazem a higienização adequada podem correr. $\mathrm{O}$ índice de desinformação sobre esse assunto foi de $58,8 \%$, e $44,2 \%$ são informados sobre $\mathrm{o}$ assunto.

As informações sobre as estratégias de comunicação e educação tendem a esclarecer e a direcionar os indivíduos sobre os riscos de contaminação. Nesta pesquisa (questão 12), 66,3\% afirmaram ter acesso a esse tipo de informação, e 33,7\% não têm.

As campanhas de prevenção de HIV, hepatites virais e outras IST são pontuais em datas específicas, com o intuito de informar as pessoas sobre o assunto. A questão 13 elencou cinco opções para conhecer qual a campanha que mais desinforma devido à falta de ações.

Tabela 1 - Campanhas de prevenção de HIV, hepatites virais e outras IST

\begin{tabular}{c|c}
\hline Campanhas pontuais de prevenção em datas específicas & Percentual (\%) \\
\hline Ministério da Saúde Federal & $86,0 \%$ \\
\hline Escolas/Universidades & $54,7 \%$ \\
\hline Secretaria de Saúde do Município & $44,2 \%$ \\
\hline Secretaria de Saúde do Estado & $41,9 \%$ \\
\hline ONGs & $25,6 \%$ \\
\hline
\end{tabular}

Fonte: Dados da pesquisa - 2018

Pode-se perceber que, na opinião dos pesquisados, as Organizações Não Governamentais fazem menos campanhas nesse sentido, ou seja, é a entidade que menos usa informações em campanhas pontuais relacionadas a estratégias de intervenção comportamental. Já o Ministério da Saúde
Federal consegue conduzir suas estratégias empregadas para esse fim.

$\mathrm{Na}$ questão 14, cinco opções foram expostas, levando em consideração a ordem do nível de desinformação a respeito do primeiro acesso à informação sobre o HIV. 
Tabela 2 - Primeiro acesso à informação sobre HIV

\begin{tabular}{c|c}
\hline Acesso à informação sobre HIV & Percentual (\%) \\
\hline Escola & $73,3 \%$ \\
\hline Família & $11,6 \%$ \\
\hline Postos e hospitais & $5,8 \%$ \\
\hline Manuais, livros e artigos & $2,3 \%$ \\
\hline TV & $2,3 \%$ \\
\hline
\end{tabular}

Fonte: Dados da pesquisa - 2018

A escola foi a opção mais citada $(73,3 \%)$ pelos entrevistados, razão por que é importante promover campanhas no ambiente escolar, onde os jovens podem absorver esse tipo de conteúdo informacional de forma mais tranquila. A família veio em seguida, com $11,6 \%$, postos e hospitais, com 5,8\%; e a internet, com $4,7 \%$. As opções como manuais, livros, artigos e a TV, com 2,3\%, revelaram o maior índice de desinformação. Se considerarmos que, para cada canal de comunicação, existe um público-alvo, são necessárias ações para promover o acesso a esses canais com menos possibilidades de uso. Portanto a informação sobre prevenção do HIV deve ser exposta em todos os canais de comunicação.

\section{CONSIDERAÇÕES FINAIS}

Nesta pesquisa, que compartilhou o questionário on-line com jovens por meio de mensagem instantânea, utilizaram-se o acesso e o meio de comunicação mais rápido a que os jovens recorrem - o WhatsApp Menssenger. A rede de relacionamentos dos jovens foi estendida a partir dos três primeiros indivíduos, "as sementes", que deram sequência ao compartilhamento do questionário caracterizando a ação da técnica de snowball. A relevância sobre a desinformação na prevenção de HIV/AIDS, que levou em consideração as ações relacionadas às intervenções comportamentais, chama à atenção para algumas ações que são consideradas na pesquisa como ausência de informações, aqui considerada como desinformação.

O nível de desinformação sobre a PrEP, demonstrado em $73,3 \%$ dos pesquisados, é alto e merece atenção, porque a PrEP, que incide no uso de ARV por pessoas não infectadas pelo HIV, pode reduzir o risco de ser infectado pelo vírus em diversas situações, como, por exemplo, nas práticas sexuais, em contextos de vulnerabilidade, nas populações com maior prevalência de HIV e em parcerias soro diferentes para HIV, parceria heterossexual ou homossexual, em que uma das pessoas é infectada pelo vírus, e a outra, não. A eficácia e a segurança da PrEP já foram verificadas em diversos estudos clínicos e segmentos populacionais, e sua efetividade foi evidenciada em estudos de demonstração (FONNER; DALGLISH; KENNEDY et al., 2016). Dentre os principais resultados dos estudos recentes, que deram impulso à aposta da PrEP como medida de prevenção ao HIV, destaca-se o fato de a eficácia dessa medida estar fortemente associada à adesão. Pesquisas apontam que, em participantes com níveis detectáveis da medicação no sangue, a redução da incidência do HIV foi de 95\% (GRANT; LAMA; ANDERSON et al., 2010). Diante do exposto, entende-se que a população pesquisada deve obter mais informações sobre o procedimento que deve ser empregado para divulgar, orientar ou usar, caso julgue necessário.

Outra ação que remete à desinformação é a ação relativa aos riscos de infecção do HIV que as pessoas que aplicam silicone industrial e hormônios não fazem a higienização adequada correm. Mais da metade dos pesquisados não teve acesso a essa informação, que é relevante quando se usam essas substâncias, porque a falta de higiene pode acarretar a incidência de infecção. A ação relativa às informações de incentivo aos jovens para realizarem o teste HIV, principalmente para os que têm uma vida sexual ativa, usam drogas injetáveis, silicone industrial ou hormônios.

O estudo indicou, ainda, que as campanhas de prevenção ao HIV, em alguns canais de comunicação, precisam ser revistas, uma vez que devem produzir efeito, levando em consideração públicos-alvo distintos, devidamente identificados e atingidos. 
No tocante ao primeiro acesso à informação sobre o vírus HIV e à doença que ele pode causar a escola vem desempenhando um papel preponderante, principalmente por ser um espaço de propagação da informação e construção do conhecimento. Já em relação aos manuais, livros e artigos sobre o assunto, percebeu-se que não foram mencionados como canais atrativos para situar esse primeiro contato, talvez seja necessário considerar que o público não está preparado ou não se sente atraído para realizar esse tipo de leitura. Quanto à TV, a informação também não recebeu adesão de primeiro contato. Isso talvez se justifique por se entender que esse tipo de informação não é comercial ou atrativa para veicular e atrair os jovens, todavia o papel de responsabilidade social e de saúde pública poderia ser abordado em dramaturgias de forma lúdica e informativa, com mais veemência e frequência, como são abordados outros assuntos.

Como achados de pesquisa, podemos afirmar que, das questões formuladas a partir das ações estratégicas de prevenção combinada do HIV, em especial, da estratégia "intervenção comportamental", que o nível de desinformação dos jovens pesquisados gira em torno de algumas situações específicas: a desinformação sobre a PrEP e sobre o uso de drogas injetáveis, o fato de usarem silicone industrial ou hormônios e a desinformação sobre o incentivo para realizar o teste a fim de se prevenir. Quanto à desinformação sobre campanhas de prevenção de HIV, hepatites virais e outras infecções, alguns órgãos, como a Secretaria de Saúde do Estado e ONGs, precisam rever suas ações, pois elas surtiram efeito nesse sentido, mas, em relação ao primeiro acesso à informação sobre o vírus, a $\mathrm{TV}$, os manuais, os artigos e os livros, precisam ser redirecionadas.

A pesquisa evidenciou que o nível de desinformação sobre a prevenção do HIV/AIDS entre os jovens pesquisados é baixo, porque, das catorze questões respondidas, apenas três apresentaram relação com a desinformação. Isso denota que, apesar do acesso à informação, o uso da informação numa perspectiva preventiva ainda é negligenciado.

\title{
IN OPPOSITION TO PREVENTIVE INFORMATION: INNACURATE INFORMTION ON HIVIAIDS PREVENTION
}

\begin{abstract}
Taking into account the access and use of information by young people today and the still relevant numbers of cases of HIV infection, the research was motivated to know the level of disinformation of young people in HIV / AIDS prevention, since, according to the Joint United Nations Program on HIV / AIDS (UNAIDS), disinformation on the subject is the major cause of young people contracting HIV. Disinformation in the counter-information condition sends an individual a state of ignorance in relation to the knowledge that would be relevant to him, with the function of guiding him and making him / her able to participate in the cognitive and evolutionary process of the informational and social chain. The objective of the research was to know the level of disinformation about HIV / AIDS prevention among young people, considering the strategies of combined HIV / AIDS prevention. Characterized as an exploratory research, the study used the snowball technique to collect data, to then generate results configured in graphs and tables for a subsequent quantitative and qualitative analysis. With the research it was possible to identify that the level of disinformation on HIV / AIDS Prevention among the young people surveyed is low, since only three of the questions answered were related to the disinformation. This leads one to believe that despite the access to information, there is negligence in relation to making use of the preventive information.
\end{abstract}


Keywords: Disinformation. Information. Prevention of HIV / AIDS.

\section{REFERÊNCIAS}

ALBUQUERQUE, Elisabeth Maciel de. Avaliação da técnica de amostragem 'Respondent-driven Sampling' na estimação de prevalências de doenças transmissíveis em populações organizadas em redes complexas. Escola Nacional de Saúde Pública Sérgio Arouca - ENSP; Rio de Janeiro: Ministério da Saúde - Fiocruz, 2009. Dissertação de Mestrado, 99p.

AQUINO, Mirian de Albuquerque. A Ciência da Informação: novos rumos sociais para um pensar reconstrutivo no mundo

contemporâneo. In: Ci. Inf. vol. 36 nº. 3

Brasília Sept./Dec. 2007. Disponível em: < http://dx.doi.org/10.1590/S010019652007000 300002>.

Acesso em: 22/11/2017.

BRASIL. Ministério da Saúde. Secretaria de Vigilância em Saúde. Departamento de Vigilância, Prevenção e Controle das Infecções Sexualmente Transmissíveis, do HIV/Aids e das Hepatites Virais. Prevenção Combinada do HIV/Sumário

Executivo/Ministério da Saúde, Secretaria de Vigilância em Saúde, Departamento de Vigilância, Prevenção e Controle das Infecções Sexualmente Transmissíveis, do HIV/AIDS e das Hepatites Virais. - Brasília: Ministério da Saúde, 2017.

. Ministério da Saúde. Departamento de Vigilância, Prevenção e Controle das Infecções Sexualmente Transmissíveis, do HIV/AIDS e das Hepatites Virais. Relatório de Monitoramento Clínico do HIV. Ministério da Saúde, Brasília: 2016.

. Ministério da Saúde. Secretaria de Vigilância em Saúde. Departamento de DST, Aids e Hepatites Virais. Pesquisa de conhecimento, atitudes e práticas na população brasileira. - Brasília: Ministério da Saúde, 2016b. 166 p.

. Ministério da Saúde. Secretaria de Vigilância em Saúde. Departamento de DST,
Aids e Hepatites Virais. Pesquisa de conhecimento, atitudes e práticas na população brasileira - Brasília: Ministério da Saúde, 2017b. Disponível em: <http://www.aids.gov.br/pt-br/noticias/seisdoencas-sexualmente-transmissiveis-em-altaentre-jovens-brasileiros-saiba-como-evita>. Acesso: 09 jan. 2018.

DEMO, Pedro. Ambivalências da sociedade da informação. In: Ci. Inf., Brasília, v. 29, n. 2, p. 3742, maio/ago. 2000.

FONNER, V. A.; DALGLISH, S. L.; KENNEDY, C. E. et al. Effectiveness and safety of oral HIV pre-exposure prophylaxis PrEP for all populations: A systematic review and metaanalysis. AIDS, 2016.

GÓMEZ, M. N. Gonzales de. Da política de informação ao papel da informação na política contemporânea. Revista Internacional de Estudos Políticos [S.1.], v. 1, n.1 p. 21-323, 1999.

GOODMAN, L. Snowball Sampling. In: Annals of Mathematical Statiscs, 32:148170, 1961.

GRANJA, E. et al. O (não) lugar do homem jovem nas políticas de saúde sobre drogas no Brasil: aproximações genealógicas. Ciênc. saúde coletiva, Rio de Janeiro, v. 20, n. 11, p 3447-3455, Nov. 2015.

GRANT, R. M.; LAMA, J. R.; ANDERSON, P. L., et al. Preexposure chemoprophylaxis for HIV prevention in men who have sex with men. N Engl J Med. 2010; 363(27):25872599. doi: 10.1056/NEJMoa1011205.

MELLO, D. F.; GONÇALVES, K. C.; FRAGA, M. F. et al. Complicações locais após a injeção de silicone líquido industrial série de casos. Rev. Col. Bras. Cir. 2013; 40(1): 037-043.

NEHMY, Rosa Maria Quadros; PAIM, Isis. A desconstrução do conceito de "qualidade da 
informação". Ciência da Informação,

Brasília, D.F., v. 27, n. 1, p. 36-45, jan./abr. 1998.

PINHEIRO. Marta Macedo Kerr. BRITO.

Vladimir de Paula Brito. Em busca do

significado da desinformação.

DataGramaZero - Revista de Informação. v.15. n 3 Dez.2014.

WALDMAN, E. A. Vigilância em Saúde

Pública, volume 7. São Paulo: Faculdade de Saúde Pública da Universidade de São Paulo, 1998. 267p.

UNAIDS (UNITED NATIONS...). 90-90-90:

An ambitious treatment target to help end the AIDS epidemic. 2014. Disponível em: < http://www.unaids.org/en/resources/ documents/2017/90-90-90>. Acessado em 26/02/2017. 